\title{
Modified superstructure fiber Bragg grating for a filter application
}

\author{
Ayser A. Hemed ${ }^{1,}$, Mayyada M. Fdhala ${ }^{2}$, Suha M. Khorsheed ${ }^{3}$ \\ ${ }^{1}$ Dept. of Physics, College of Education, Mustansiriyah University, Baghdad, IQ \\ ${ }^{2}$ Distinguished girls' secondary school, second Karkh Directorate of Education, Baghdad, IQ \\ ${ }^{3}$ Dept. of Physics, College of Science, University of Al-Nahrain, Baghdad, IQ \\ *Corresponding author: ayser.hemed@uomustansiriyah.edu.iq
}

\begin{abstract}
Performance of a modified superstructure fiber Bragg grating is carried out by; simulation using a Matlab and then by optisystem7. Results for these two simulations are compared with an experimental investigation for three special FBGs in addition to a standard one. These four FBGs have one, two, three, and four regions. For each region, Bragg wavelength is increased around $6 \mathrm{~nm}$, with constant spacing between every two regions. Laser reflectance (R) and transmittance $(\mathrm{T})$ spectra and their corresponding number of peaks and their bandwidths are observed and analyzed in a constant temperature and strain.

Results for this investigation indicate enhancement for the modified FBG to be an effective filter that can be employed for communication as well as sense. Filtering the transmitted signals could be satisfied in a simple and more efficiency than the traditional device.
\end{abstract}

Keywords: Birefringence; Bragg wavelength; chaotic dynamics; couple mode theory; optical fiber filter; superstructure fiber Bragg grating.

\section{Introduction}

Fiber Bragg Grating (FBG) based sensing technique, is a well-known technique, in which a little and small bandwidth for reflectance spectrum is enough (Singh et al, 2017). While in the nonlinear and chaotic dynamics field of study, increased chaotic bandwidth is very necessary for large data uploading via the generated chaos (Hemed, 2011). Noting that, in the first application, the $\mathrm{T}$ spectrum is considered, while in the second, the $\mathrm{R}$ spectrum is used to optically excite laser instabilities before employing it for encryption. In which optical injection is another option (Hemed \& Abbas, 2020). The latter is based on identical chaos synchronization with high cross-correlation (Joarder \& Omar, 2013) value between the two constructed chaotic units i.e., units that oscillate identically with higher-order nonlinearity regimes (Bayat, 2015). In these two applications, FBGs have developed into important components (Othonos et al., 1999). According to their specific parameters, FBGs are capable to classify. In case of their period, FBGs are divided into FBG (ordinary) and long-period grating (LPG) (Alemohammad, 2018). While in the case of index axial modulation FBGs are uniform and superstructure. According to its slit's inclination angle with fiber axis, tilted FBG is another type. Chirped and sampled FBGs are also more types (Amiri et al., 2018). 
For all the above types, FBG couples light from the forward to the backward guided modes furthermore cladding modes. Thus, Bragg reflection construct and many small dips in transmission at shorter wavelength side of the Bragg wavelength rises. For the LPG, which has a period of hundreds of micrometers, couples light from the forward guided mode to the forward cladding (leaky) modes, resulting in attenuation bands in the transmission spectrum.

Another application for commercial investigators has attractively improved FBGs array spectrometry detection. In this settlement, a broadband light (extended wavelengths) source is required to illuminate the array, and a spectrum analyser or spectrometer is used to combine the deflected spectra (Tosi, 2017).

Superstructure FBG (SFBG) is a periodically modulated FBG, which introduces counter directional guided mode-coupling at a series of regularly separated wavelengths results in a comb-like reflection spectrum (Eggleton et al., 1994) (Chow et al., 1996). This property of the SFBG has been fully analyzed using the coupled-mode theory (Raphael et al., 1997). Recently, Bo Pang et al. (Panga et al., 2020) modified the coupled-mode equation of superimposed coated LPG for which many applications have been demonstrated including surrounding refractive index and temperature measurements. Some examples of the applications of SFBG are comb filters for constructing tunable distributed Bragg reflector (DBR) fiber lasers (Ibsen et al., 1995) and multi-wavelength fiber lasers (Chow et al., 1996); multi-channel dispersion compensator in wavelength-division-multiplexing (WDM) systems Zhang et al., (2002). Due to their property of low young's modulus, polymer optical FBGs meets a lot of attention over the last two decades for sensing applications. These applications include; strain, temperature, the potential of Hydrogen $(\mathrm{PH})$ for water or health conditions and measuring relative humidity (Woyessa et al., 2020); stress-induced Birefringence (Hemed, 2005), and controlling chaotic anti-synchronization via FBG sensor (Hemed et al., 2021).

It is worth noting that the key advantage of SFBG, when used as a sensor, is that it possesses both the characteristics of FBG and LPG in a single optical fiber (Sengupta et al., 2016). Photolithography is described for design a refractive index FBG in photo-sensitive fiber by using a special phase mask grating made of $\mathrm{SiO}_{2}$ glass (Hill et al., 1993). This method is based on an ultraviolet (UV) laser source. Several laser sources can be used, depending on the following: type of fiber used for the grating, type of grating, and intended application. However, restraining a fiber during grating fabrication alters the FBG wavelength in the relaxed state. It is also possible to multiplex several gratings in the same location to form more type gratings (Kashyap, 2009).

It should be noted that the Bragg wavelengths of all multiplexed gratings written at the same location shift to longer wavelengths as each grating are superimposed. The shift in the wavelength of the gratings is dependent on the overall change in the index of modulation, resulting in a change in the period averaged $n_{\text {eff }}$ of the mode in the fiber. A shift of $\Delta \lambda_{B}$ in the Bragg wavelength $\lambda_{B r a g g}$, as the UV induces change $\delta n$ increases, can be given as: 
$\Delta \lambda_{B}=\lambda_{B} \frac{\eta \delta n}{n_{e f f}}$

Where $\eta<1$, is the overlap of the guided mode and the distribution of the refractive index modulation. Thus, when a grating is superimposed on an already-written grating, both gratings move to longer Bragg wavelengths (Hill et al., 1978).

Light propagating through the core of the fiber would, because of these periodic perturbations, be back-reflected along with the core at a Bragg wavelength, $\lambda_{B}$ with a certain line width. The Bragg wavelength would depend on the period $(\Lambda)$ of the perturbations and effective index $\left(n_{e f f}\right)$ of the optical fiber.

$\lambda_{B}=2 n_{e f f} \Lambda$

SFBGs are special FBGs that have periodic index modulations distributed across a segment of fiber in the fiber core. This is effectively a secondary index profile modulation in a length of uniform FBG with a much longer pitch than the Bragg grating itself. The reflectivity characteristics of such structures are predicted to present reflectance peaks from each spatial Fourier component of the index perturbation.

The constant periodic SFBG includes a series of grating then non-grating segments with equal lengths. The grating segments act as normal FBG and result in several reflectance spectrum peaks in the side of reflectance (deflection) (Sengupta, 2015). These peaks are located at different frequencies with different reflectivity and other higher-order Fourier components (Eggleton et al., 1994). Hence, despite being a single grating, the effect of an SFBG is similar to several smaller FBGs placed close to each other. From on hand, operating by laser diode, FBGs (or SFBGs), themselves are not sensitive to perturbations in ambient refractive index (RI) unless they are inscribed in an etched or a tapered fiber (Eggleton et al., 1994). On the other hand, for higher power lasers, amplified spontaneous emission and thermal effect management is a major challenge in high-power solid-state lasers oscillator and amplifier design (Cheng et al., 2018).

The spectral profile of a pulse passing within a traditional (one region) Bragg grating, as mentioned earlier, can be obtained from the modes and reversed modes interplay based on coupled equations starting from the nonlinear Schrödinger equation (NLSE) for signal propagating inside the optical fibre i.e.

$$
\begin{aligned}
& \frac{\partial u^{+}}{\partial z}-i\left[k-\frac{1}{2}\left(\Delta \beta-\frac{\partial \varphi(z)}{\partial z}\right] u^{+} \exp ^{-i[\Delta \beta z-\emptyset(z)]}=0\right. \\
& \frac{\partial u^{-}}{\partial z}+i\left[k+\frac{1}{2}\left(\Delta \beta-\frac{\partial \varphi(z)}{\partial z}\right] u^{-} \exp ^{i[\Delta \beta z-\emptyset(z)]}=0\right.
\end{aligned}
$$

Where, $\mathrm{k}$ is the coefficient of coupling between incoming $\left(u^{-}\right)$and outgoing $\left(u^{+}\right)$two modes that passing through one region FBG, $\Delta \beta$ is the phase mismatching, which is given as; $\Delta \beta=$ 
$\left(w-w_{B}\right) \cdot \frac{n_{e f f}}{C}, n_{e f f}$ is core effective index, C speed of light at Bragg wavelength given by equation (2), in calibrating with which one can determine Bragg frequency; $w_{B}=\pi C / n_{\text {eff }} \Lambda$, and $\emptyset$ is the grid period chirp and it vanishes for the uniform FBG, (Filho et al., 2019).

According to the weak coupling coefficient limit, the grating reflectivity can be approximated as the Fourier transform which depends on both coupling coefficient distribution along the grating and the detuning. As a result of Every Fourier component contribution, reflectivity peak is constructed in the reflection spectrum of the SFBG, and the wavelength spacing of the Bragg peaks is given by:

$\Delta \lambda=\frac{\lambda_{B}^{2}}{2 n \Lambda}$

Where, $\lambda_{B}$ is Bragg wavelength and $\Lambda$ is the period of low-frequency envelope (Jinsong et al., 2004).

\section{Experimental set up}

This work is mainly divided into two parts, theory as a simulation (OptiSystem 7) and experimental part. Both two parts are configured for calculating the following parameters: characteristics of reflected and transmitted spectra. These characteristics are based on full width at half maxima (FWHM) (pulse duration) and center wavelength drawn from OSA and number of peaks drawn from radio frequency spectrum analyzer (RFSA), both observations were carried out separately from reflected then transmitted signals. Grating's variables including length of grating, the period of the grating, and effective refractive index, are presented in Table (1). Change in the number of regions of the grating is the main factor affecting this study. This investigation was repeated both theoretically (by simulation) and experimentally in the laboratory.

\subsection{Simulation part}

Excluding the effect of both temperature and stress-induced birefringence, several configurations (shown in Figure 1) were investigated, with all considered parameters are included in Table 1. The first one (Figure 1A) is a uniform one region FBG sensor, subjected to $1550 \mathrm{~nm}$ wavelength light. Both deflected and transmitted signals were observed by an alloptical spectrum analyzer (OSA), RF spectrum analyzer (RFSA) and, oscilloscope (DSO). FBG parameters are given in Table 1. The light source is chosen to be the same for all simulation configurations (and experiments as well). For all cases Figure 1(ABC) light is directed from LD is passing through a frequency modulator (FM) for external modulation, which is mixing with external frequency signal. Light that emerges from the modulator is going to be split into two directions. One of them (10\%) is for sampling, while the remaining $(90 \%)$ is for the FBG investigation purpose. Deflected light from the standard FBG goes to another detection. The same action, with additional detection, is carried out for the transmitted light. 
The same procedure is followed with the remaining superstructure FBGs, i.e., both filtered and deflected signals observed by; OSA, RFSA, DSO. The difference, in this case, is with the deflected parts combination. Where we have more than one deflection (three in the three regions FBG and four in the four regions FBG).

This simulation is carried out in parallel to a mat lab program considering the solution of mode-coupling theory (MCT). In which the grating (condition) equation for index perturbation ( $\left.n_{\text {eff }}\right)$ of the optical fiber guided mode is given in equation (2). The program code of FWHM was modified from reference; (Mohammed et al., 2018).

\subsection{Experimental part}

As shown in figures $2 \mathrm{~A}, \mathrm{~B}, \mathrm{C}$, three setups were considered in this work. To calculating the effect of grating regions number on the efficient of FBG optical power meter is used as well as the OSA amplitude counts level measurements for detected light. Standard FBG and two types of modified SFBG were designated. The first has three regions and the second has four. Parameters for all those FBGs (with three and four grating regions) are given in Table 2. CW laser diode (LD) model: (SAT-3E05) is used as a light source. Optical fiber for these FBGs was manufactured from acrylate- single mode for standard FBG, and acrylate (SMF-28e) for SFBG.

Table 1. Parameters (for experimental and simulation) for the investigated SFBG.

\begin{tabular}{cc}
\hline Effective refractive index( $\left.\boldsymbol{n}_{\text {eff }}\right)$ & 1.444 \\
\hline Bragg wavelength $\left(\boldsymbol{\lambda}_{\boldsymbol{B}}\right)$ & Uniform FBG: $1539.185 \mathrm{~nm}$ \\
[manufactured specially] & $1530.398 \mathrm{~nm}$ \\
ID: 180119-02 and 180119-03. & $1536.478 \mathrm{~nm}$ \\
& $1542.169 \mathrm{~nm}$ \\
SFBG (Four grating regions): & $1530.336 \mathrm{~nm}$ \\
Index difference between core and & $1536.506 \mathrm{~nm}$ \\
Radius of core (a) & $1542.289 \mathrm{~nm}$ \\
[Designed and manufactured FBG] & $1548.045 \mathrm{~nm}$ \\
Grating lengths $(\mathbf{L})$ & 0.0036 \\
(Mohammed et al. 2018$)$ & $4.1 \mu \mathrm{mm}$ \\
Number grating regions in FBG $(\mathbf{N})$ & $1,2,3,4$ \\
\hline
\end{tabular}



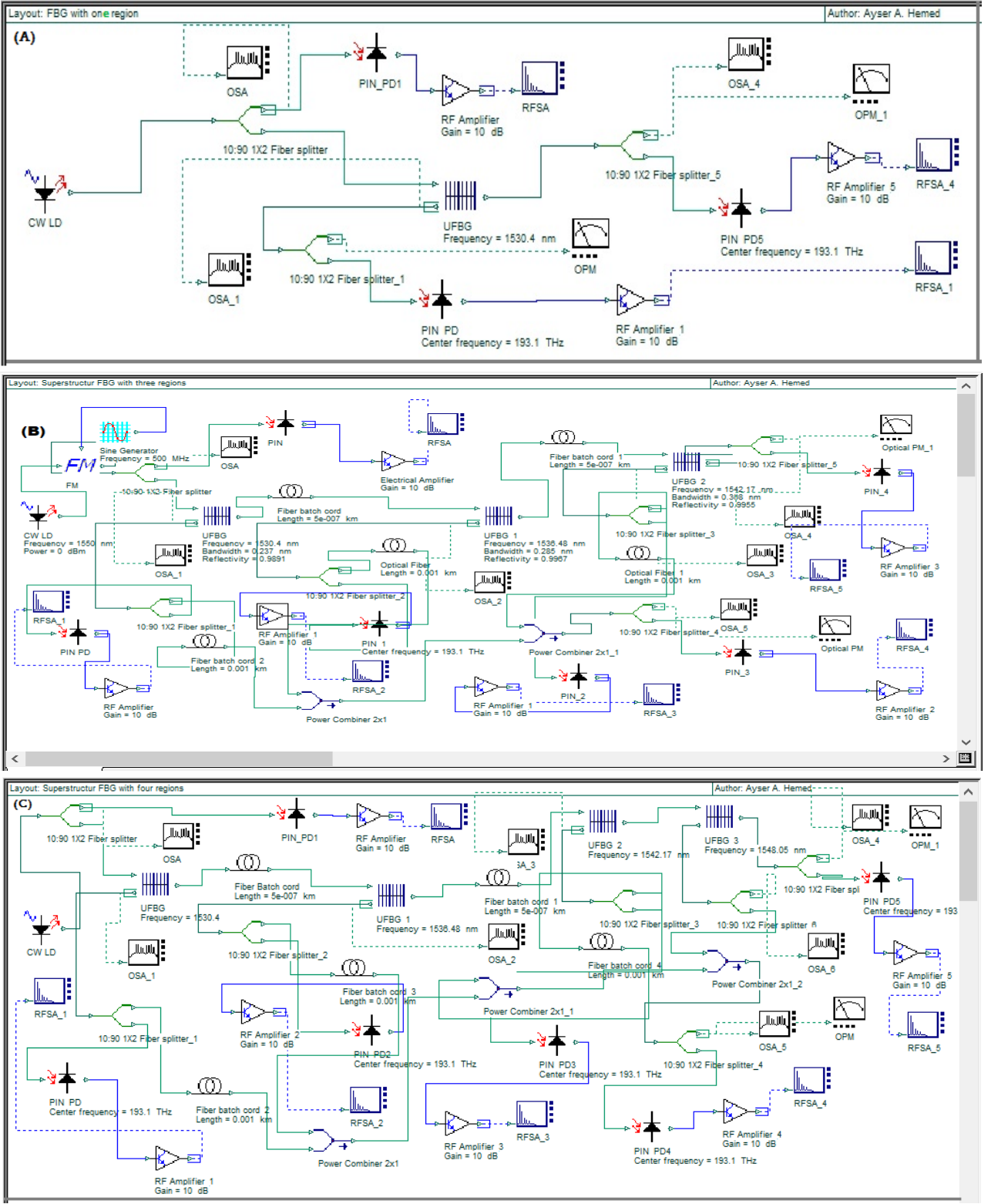

Fig. 1. Simulation diagram for deflected and transmitted laser spectra vis FBG;

(A) Standard one region, (B) Three regions, and (C) four regions. 2

Table 2. Details for the experimental modified SFBG sensor.

\begin{tabular}{|cccc|}
\hline $\begin{array}{c}\text { Reflectivity } \\
(\%)\end{array}$ & $\begin{array}{c}\text { Bandwidth } \\
(\mathbf{n m})\end{array}$ & $\begin{array}{c}\text { Peak reflectance Wavelength } \\
(\mathbf{n m})\end{array}$ & $\begin{array}{c}\text { Number of } \\
\text { regions }\end{array}$ \\
\hline 94.25 & 0.176 & 1539.185 & One region \\
98.91 & 0.237 & 1530.398 & \\
99.67 & 0.285 & 1536.478 & Three regions \\
99.55 & 0.308 & 1542.169 & \\
99.06 & 0.242 & 1530.336 & Four regions \\
99.51 & 0.275 & 1536.506 & \\
99.58 & 0.312 & 1542.289 & \\
99.61 & 0.311 & 1548.045 & \\
\hline
\end{tabular}




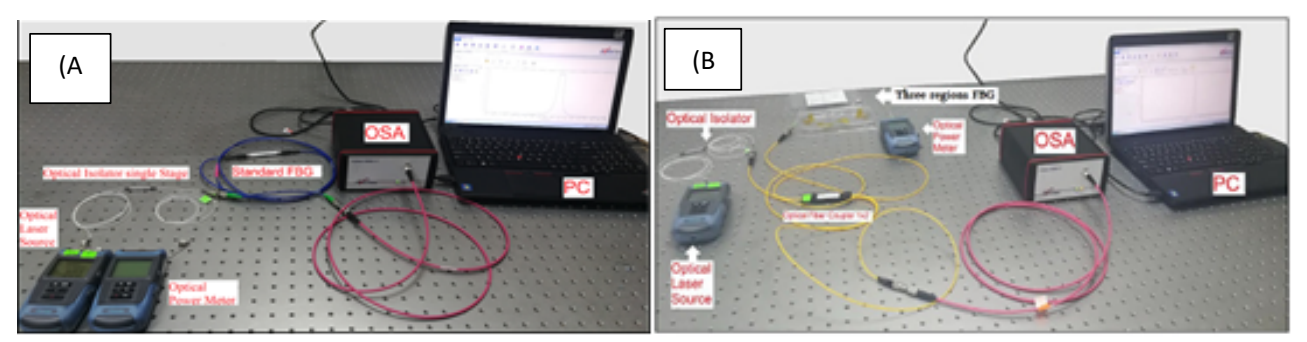

Fig. 2. FBG measurements experimental setup. (A) standard, (B) Three regions.

It is worth mentioning that the specifications of the SFBG sensor were determined theoretically then sent to the manufacturer according to the specifications that satisfy the desired application for the study.

Figure 2 shows configuration setups for direct transmitted power measurement; (A) standard, (B) Three regions (four regions are the same as part (B)), FBGs, respectively. Figure (3) shows the setup for reflected spectrum observation. Conditions with which the experiment was run, laser operating temperature was roughly constant at $\left(25^{\circ} \mathrm{C}\right)$

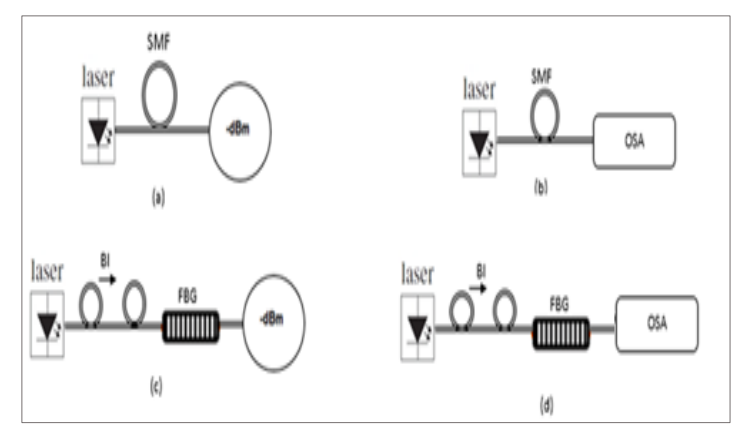

Fig. 3. Experimental set ups for measuring; (a) laser power, (b) spectrum, (c) FBG transmitted power, and, (d) FBG transmitted spectrum.

and the length of the grating was constant according to Table (1), keeping FBG temperature and stress unchanged. The only parameter that changed was the number of the grating regions. Direct measure for input and output optical power incident and passing via the FBG is done by using the optical power meter. Laser source output power reads (-6.2dBm). Efficiency calculation follows the relation (Singh et al., 2017):

$$
\eta(\%)=\frac{p_{\text {out }}}{p_{\text {in }}} \times 100 \%
$$

Where; $p_{\text {out }}$ is the output power, $p_{\text {in }}$ is the input power.

Power unit's transformation from $\mathrm{dBm}$ to Watt follows the relation (Chapra, 2012)

$$
m W=10^{\left(\frac{d B m}{10}\right)}
$$




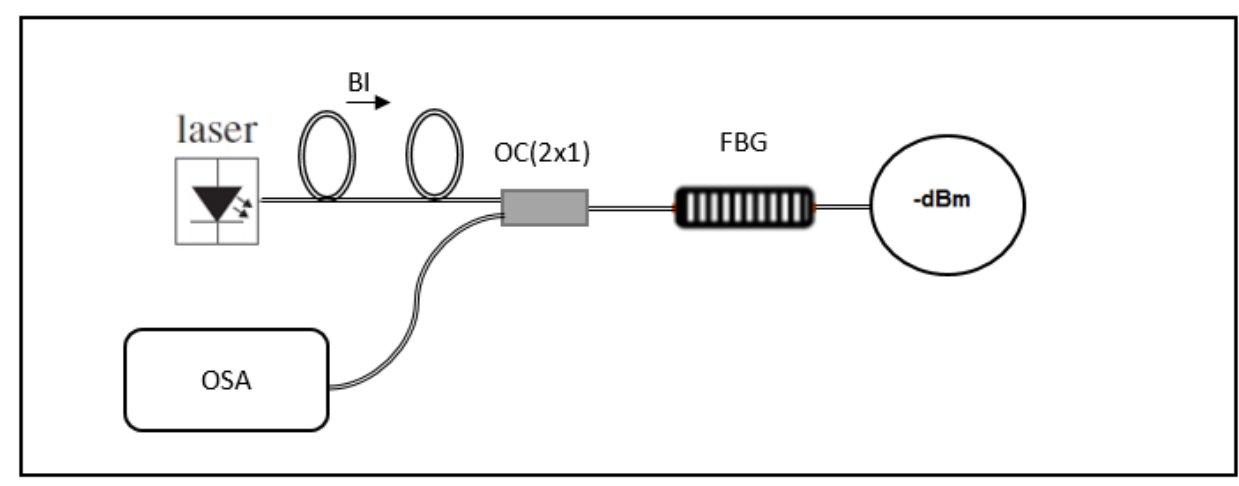

Fig. 4. Setup for reflected spectrum observation

The other method for calculating the efficiency is calibrating is by considering the spectrum counts scale for the AvaSpec (software version 8) input and output spectrum. The deflected spectra of the FBG sensors were determined experimentally by the setup which is shown in Figure (4). The same Figure can be used to observe the transmitted spectrum i.e., the laser source is connected into the beam isolator $(\mathrm{BI})$ input, (to prevent laser emission from generated instabilities due to optical feedback (back-reflected power) which may destroy the laser spectrum). The signal is then sent into one arm of a $2 \times 1$ 50:50 fiber optical coupler (OC) such that one of these ports injects the laser optical power into the FBG input, and the other receives the back-reflected beam. After passing via the FBG, optical power will transmit to the OSA.

All considered types of FBG that are considered in this work are connected separately as in Figure 3 to study their spectra.

Using the uniform superstructure FBG in optical communications is an interesting approach. In which the transmitted signals could be filtered. This is possible by locating it within a fiber transmission line to reject specific wavelengths. (Zhao, 2001), this technique is called active filtering. The deflected part of the signal is also can be used to introduce instability to the laser to generate the coherence collapse. Another type depends on the ability to fine-tuning the filter mentioned by Campanella et al., (2018).

Several configurations setups were investigated for both transmitted and reflected spectrum to check the best filtering efficiency from them with considering the efficiency.

\section{Results and Discussion}

In addition to the Matlab program simulation, OptiSystem 7 was also employed to calculate SFBG (3 and 4 grating regions) parameters for the estimated reflectance spectrum; this is to include the MCT. Standard device datasheet parameters were also included in those two simulation methods. Additional grating variables, which are length of the grating regions, grating period, and $\left(n_{e f f}\right)$ were adopted from reference mentioned in Table (1). Although an FBG spectrum has several contributions, such as $\lambda_{B}$, bandwidth, reflected (and transmitted) intensity, etc. Measuring these attributes with a high-resolution requires special instruments such as high-resolution OSA. In industrial applications, a tunable filter is frequently used for FBG response and the $\lambda_{B}$ is the sensing parameter that can be obtained properly. Since the $\lambda_{B}$ is sensitive to temperature and strain changes, it is impossible to differentiate the effects of temperature and strain using it alone, which is the main challenge in such studies. Currently, 
this study focuses on calculations with a constant temperature and stress, i.e. photosensitivity is excluded temporally to be able to design the modified FBG.

Figure (4) shows reflected spectrum calculations for SFBG with three grating regions that have the following Bragg wavelengths; (upper Figure part) $\lambda_{B 1}, \lambda_{B 2}, \lambda_{B 3}=1530.398,1536.478$ and $1542.169 \mathrm{~nm}$, respectively, and for the four grating regions; (lower Figure part) $\lambda_{B 1}, \lambda_{B 2}, \lambda_{B 3}, \lambda_{B 4}=1530.336,1536.506,1542.289$ and $1548.045 \mathrm{~nm}$. For the three regions, FBG resulted in reflection wavelength spacing is about $6.5 \mathrm{~nm}$ between the green and red, and $6 \mathrm{~nm}$ between the red and blue. Similarly, for the four regions FBG, it equals, 6.5, 4, 4, and $3.5 \mathrm{~nm}$, respectively. These results agree with (Alemohammad, 2018) except value No.1. Another observation for these deflected peaks is that they are equal in intensity. This is not possible, but if we consider the small scale (around the inverse thousand) we can have

Understood that they are near equals. Addition to that, these peaks resulted from forward and backward interacting modes which may give them a creative linear interference gain.
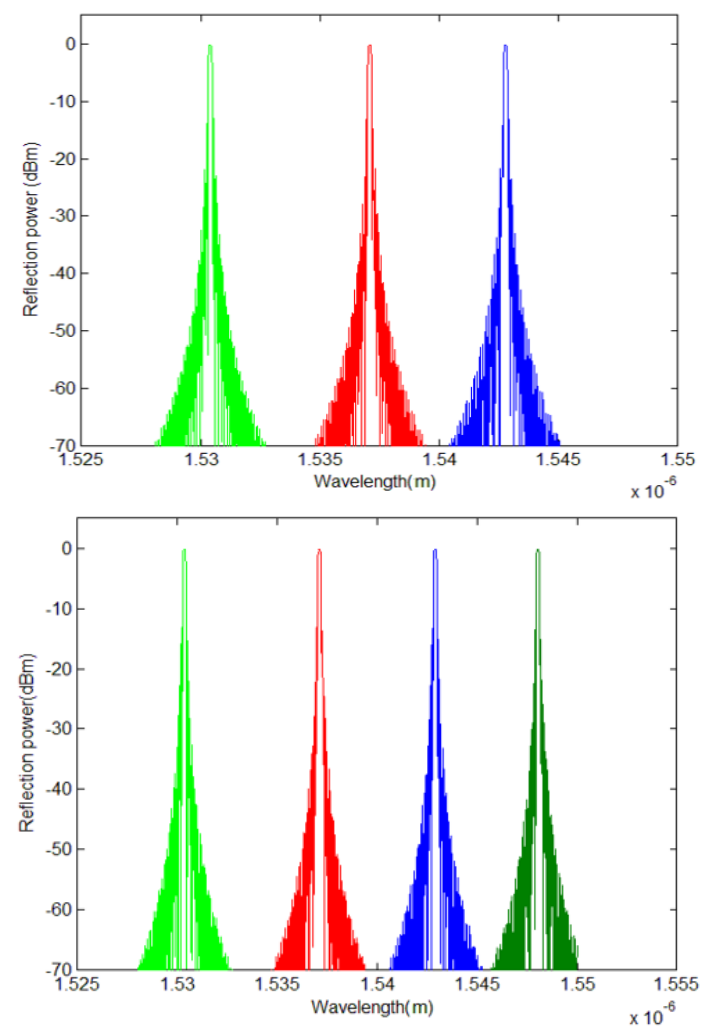

Fig. 5. Reflection spectra for Matlab simulation part. (A) 3 and (B) 4 regions

Reflectivity for the wavelength for different values of grating regions assuming that $n_{\text {eff }}=$ 1.444 and $\mathrm{L}=1 \mathrm{~cm} . \lambda_{B}$ appears a sharp peak reflected from grating regions. These peaks satisfy Bragg condition equation (2). Bragg wavelength and bandwidth are both increased with increasing of grating regions for SFBG as shown in Table (3). According to these results, the FWHM value increases linearly with increasing of Bragg wavelength for each new FBG region. The power efficiency of the spectrum of transmitted FBG sensors for SFBG sensor calculated by using equation (6). Experimentally, measured laser transmitted spectrum parameters relative to SFBG are given in Table (4). Results peak wavelengths, output power, intensity, the efficiency of power, and the bandwidth (FWHM) for observation of SFBG with 
four grating regions. As shown in this Figure, there exists a non-negligible broadening in peaks of the optical spectra for experimental results. This broadening can be represented as a wavelength variation with the axial position of grating i.e. $(d \lambda / d z)$. The last value varies for each reflection and varies than transmitted signals all concerning to the incident signal.
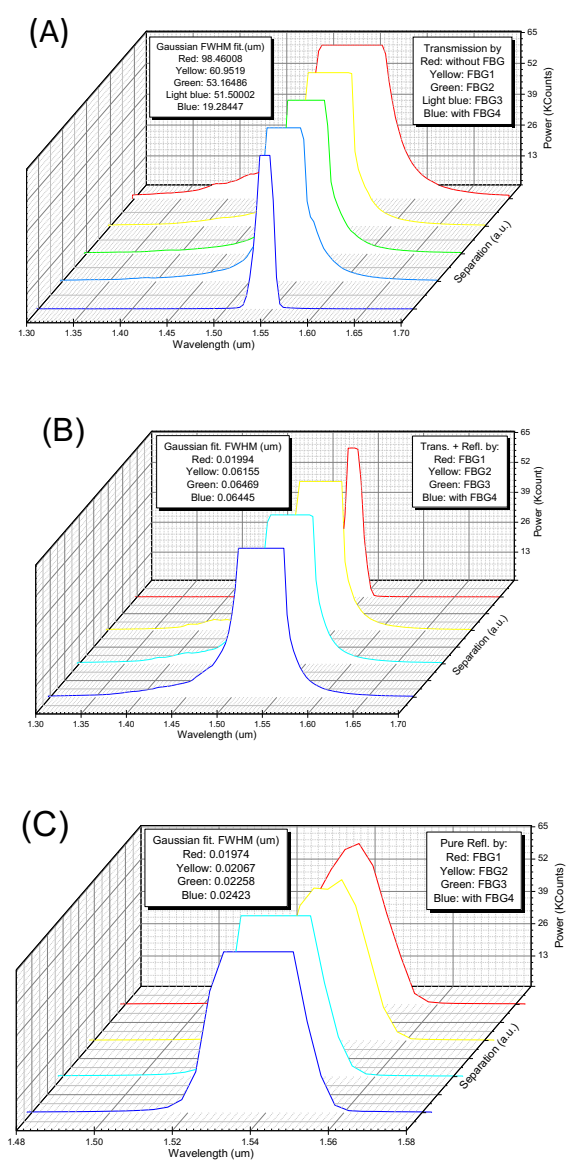

Fig. 6. Experimental results for; (A) transmission, (B) mixed transmitted and reflected, and (C) pure reflected signal into FBGs with number of regions mentioned inside the Figure separately.

Noting that according to theoretical background based on linear (on random) chirped gratings treatment of mode lock theory, the value of $(d \lambda / d z)$ can take a positive or negative value. This broadening can be also considered as a right (or left) shift from designated Bragg wavelength to an experimentally measured spectrum. Practically, this shift is interpreted as a time delay in this signal after hitting or passing via FBG and can be determined by the product of the wavelength spacing and the dispersion introduced by the FBG medium. In association with this measured wavelength, degradation in signal is also observed as a result of mode interactions during pass or deflection between different existing modes.

According to Bragg wavelength given in Tables 3, 4, 5 one can found that these SFBGs may also consider as chirped Bragg gratings. In which the period of a grating varies linearly with fiber axial position. This means that grating reflects different wavelengths at different points along this length. In the case of chirped Bragg gratings, signals can be modeled by the coupledmode theory. The refractive index for such gratings can be expressed as a function of $\mathrm{x}, \mathrm{y}, \mathrm{z}$ 
fiber coordinate components, such that the original index is $\mathrm{n}(\mathrm{x}, \mathrm{y}, \mathrm{z})$ must be added to the perturbed index change, which is now a function to cosine function. The cosine term should contain both instantaneous Bragg wavelength and phase of the chirped grating. Noting that the linear chirp is easier to solve than the arbitrary chirp.

Table 3. FWHM simulation of different grating regions number of FBGs.

\begin{tabular}{ccc}
\hline $\begin{array}{c}\text { number of } \\
\text { regions } \\
\text { for FBGs }\end{array}$ & $\begin{array}{c}\text { Bragg } \\
\text { wavelength } \\
\text { (nm) }\end{array}$ & $\begin{array}{c}\text { FWHM } \\
\text { (nm) }\end{array}$ \\
\hline SFBG & 1530.398 & 0.214 \\
(three regions) & 1536.478 & 0.255 \\
& 1542.169 & 0.305 \\
SFBG & 1530.336 & 0.234 \\
(four regions) & 1536.506 & 0.256 \\
& 1542.289 & 0.308 \\
\hline
\end{tabular}

Results indicate that the deflection is shifted toward the longer wavelengths. Part of the light that satisfies Bragg condition reflected from the grating, while wavelengths grater is not, instead transmit, so when there exists more than one grating regions, with different conditions, different effective refractive index $n_{\text {eff }}$ will exist as well, i.e. more wavelengths will reflect instead of a unique one. Therefore, experimentally spectral analysis shows effective refractive index $n_{\text {eff }}$ will exist.

Table 4. The transmitted spectrum parameters relative to SFBG

\begin{tabular}{cccccc}
\hline Fiber Type & $\begin{array}{c}\text { Efficiency of } \\
\text { Power (\%) }\end{array}$ & $\begin{array}{c}\text { Output } \\
\text { Power } \\
(\mathbf{d B m})\end{array}$ & $\begin{array}{c}\text { FWHM } \\
\mathbf{( n m )}\end{array}$ & $\begin{array}{c}\text { Intensity } \\
\text { (Counts) }\end{array}$ & $\begin{array}{c}\text { Peak } \\
\text { Wavelength } \\
\boldsymbol{\lambda}_{\boldsymbol{p}}(\mathbf{n m})\end{array}$ \\
\hline $\begin{array}{c}\text { Single mode fiber } \\
\text { SFBG }\end{array}$ & 95.50 & -6.4 & 100.141 & 66350.0 & 1496.847 \\
$\begin{array}{c}\text { (Three regions) } \\
\text { SFBG }\end{array}$ & 72.76 & -7.78 & 48.88 & 67292.52 & 1547.014 \\
\begin{tabular}{c} 
(Four regions) \\
\hline
\end{tabular} & 56.35 & -8.89 & 24.83 & 66667.59 & 1536.317 \\
\hline
\end{tabular}


Table 5. The reflected spectrum parameters relative to SFBG.

\begin{tabular}{ccccc}
\hline Fiber Type & $\begin{array}{c}\text { Bragg } \\
\text { Wavelength } \\
\boldsymbol{\lambda}_{\boldsymbol{B}}(\mathbf{n m})\end{array}$ & $\begin{array}{c}\text { Intensity } \\
\text { (Counts) }\end{array}$ & $\begin{array}{c}\text { FWHM } \\
(\mathbf{n m})\end{array}$ & $\begin{array}{c}\text { Efficiency } \\
\text { (Intensity)(\%) }\end{array}$ \\
\hline $\begin{array}{c}\text { SFBG3 } \\
\text { (three grating }\end{array}$ & 1527.02 & 69507.44 & & 99.31 \\
regions) & 1534.89 & 65930.88 & 24.88 & 97.98 \\
& 1542.03 & 67924.79 & & 97.05 \\
SFBG4 & 1530.24 & 66363.47 & & \\
(four grating & 1536.67 & 65597.18 & & 99.54 \\
regions) & 1542.74 & 64993.87 & 27.29 & 98.40 \\
& 1545.59 & 68015.33 & & 97.49 \\
\hline
\end{tabular}

As well, i.e., more wavelengths will reflect instead of a unique one. Therefore, experimentally spectral analysis shows more than one peak i.e., linear dependence with grating number of regions. SFBG with three grating regions and four regions gives more reflected wavelengths that can be used in different applications such as sensing and communications which can send more data.

Table (5) shows that Bragg wavelength which is measured if compared with wavelengths of datasheets for the used FBG listed in Table (2), with peaks close to each other. Bandwidth is also increased for reflected spectrum for different region number grating regions.

Including MC between the forward core mode and backward core mode and the core mode to both forward-propagating and backward-propagating cladding modes of first azimuthal order (fundamental lasing mode). Circular symmetric cladding modes of odd order have a similar electric field with core mode, then have large overlapping integrals. Then, the coupling between them would be more probability efficient and should be taken into account in the calculations. To formulate the transmission characteristics of SFBG, a matrix of an FBG section must be formulated with that of a non-grating or phase shift section for a specific cladding mode.

Measured power intensity efficiency shows decreased value compared with the transmitted spectrum of SFBG sensors.

The laser spectrum which is used has wide bandwidth $(100.141 \mathrm{~nm})$ for length $(1450-1600)$ $\mathrm{nm}$ around $1550 \mathrm{~nm}$. The bandwidth decreases in transmitted and reflected for SFBG that is due to internal built-in FBG sensor multi regions. This result indicates a higher passband in comparison with our earlier results in ref. (Fdhala et al., 2020), in which measurements were based on a standard FBG with one grating regions.

On one hand, if we consider the experimental study carried out by ref. (Khorsheed et al., 2019), with the present results, it is worth mentioning that increasing the FBG regions gives rise to filtering process efficiency in cost of efficiency lowering. This lead to the ability of such 
a filter to stimulate a rich laser diode chaotic dynamics associated with the optical feedback and injection, such as that published in the reference (Hemed et al., 2020). Where such a technique gives the facility to operate the laser in multi-stability states and this will give it more and more tune-ability for different applications with the same laser device. Additionally, its capacity to select each one (or both) from the back-reflected optical power and then redirect its radio frequency as a current to be re-injected into the laser bias. This possibility represents a modified self-electro optic effect device (SEED) laser. While insertion of the later current is another possibility with the existence of an electro-optic modulator, such as that mentioned in refs. (Macrae et al., 2020; Leng et al., 2004).



Fig. 7. Optical spectra for incident, deflected and transmitted signals, 3 regions FBG.

Simulation for optical spectra results shown in Figure 5 for reflected spectrum shows weaker pulse due to matching between laser operating center wavelength and regions Bragg wavelengths variation. In case of modulation, D.C. level increased, then these spectra become higher intensity than without modulation, as shown in Figure 6.



Fig. 8. Optical spectra associated with $100 \mathrm{MHz}$ modulation in a standard FBG.

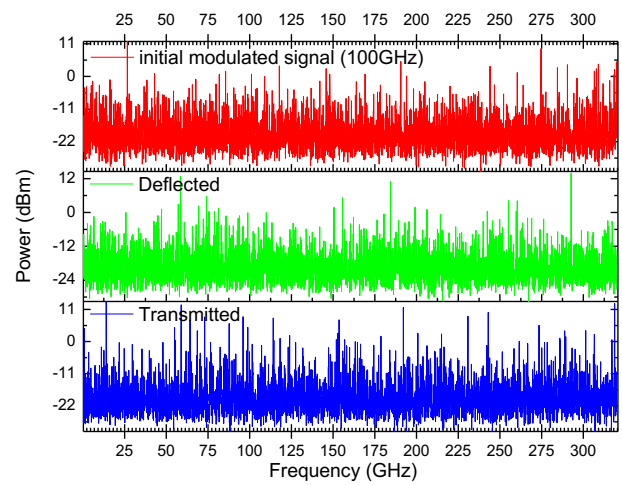

Fig. 9. RF spectra associated with $100 \mathrm{GHz}$ modulation in a standard FBG. 
For the same simulation measure, the RF spectra is shown in Figure 7. The program measured no of peaks above $0 \mathrm{dBm}$ are: 22, 17, 22, 19, 33, 28.

Simulation result for optical spectra to the case of three grating regions FBG with modulation of $100 \mathrm{GHz}$ gives the optical spectra shown in Figure 8.

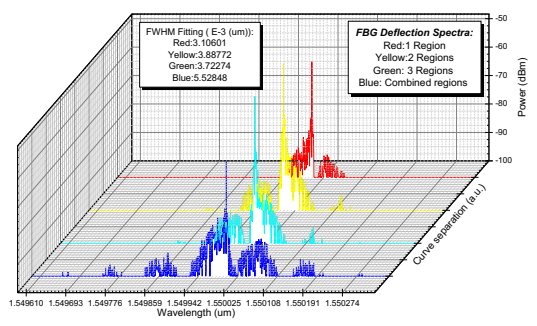

Fig. 10. Optical spectra for standard 3 regions FBG with $100 \mathrm{GHz}$ modulation

For the case of four regions FBG, optical and RF spectra is shown in Figures 9 and 10.



Fig. 11. Optical spectra associated with deflection, for each region separately with modulation in 3 regions FBG.

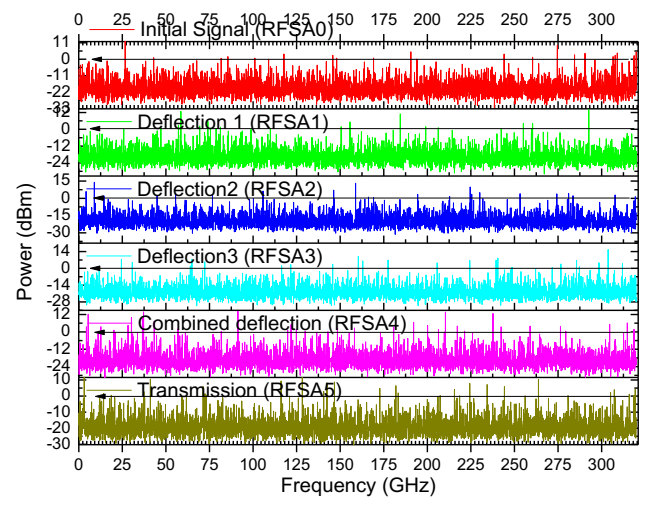

Fig. 12. RF spectra associated with incident, deflected and transmitted signals for 4 regions FBG.

As shown in Figure (9), overall reflected peak wavelength is $1.54836 \mathrm{~nm}(-141.00 \mathrm{dBm})$ which is a combination from two separately wavelengths; $1.54921 \mathrm{~nm}(-83.71 \mathrm{dBm})$ and $1.54950 \mathrm{~nm}$ 
$(-69.58 \mathrm{dBm})$. The loss makes reflected peak amplitude to be more attenuated comparable to source, and transmitted peaks. This is due to coupling between the forward core mode and forward cladding mode that gives rise to attenuation. Addition to that, the majority of light in principle transmits the FBG, this makes the red spectrum has a higher peak amplitude than the reflected one.

\section{Conclusions}

The observed bandwidth for the reflected spectrum for the four grating regions is wider than that observed with the three grating regions. The behavior of the signal is affected by several parameters, such as negative and positive dispersion, forward and backward modes during multiple reflections in each FBG region. Variation of Bragg wavelength for each region inside the same device makes modes interplay more complicated than that given in MCT. Mismatching between simulation and experimental results is ascribable to many factors, such as considering the laser source an ideal emitter in simulation versus experiment, the FBG medium maybe impure, which gives rise to different types of dispersion effects to work.

\section{ACKNOWLEDGEMENTS}

The authors would like to thank Mustansiriyah University, (www.uomustansiriyah.edu.iq) Baghdad - Iraq for their support in the present work.

\section{References}

Alemohammad, H. (2018). Opto-Mechanical fiber optic sensors; Research, technology, and applications in mechanical sensing - chapter two, P.p 27-47. Elsevier Inc.

Amiri, I.S. et al. (2018). Introduction to photonics: principles and the most recent applications of microstructures. Journal of Micromachines 9, 452.

Bang, O. (2020). Enhanced pressure and thermal sensitivity of polymer optical fiber Bragg grating sensors. Science Direct-Elsevier, Journal of Optics and Laser Technology. 130.

Bayat, M., Pakar, I., \& Bayat, M. (2015). Nonlinear vibration of mechanical systems by means of Homotopy perturbation method. Kuwait J. Sci. 42 (3) pp. 64-85.

Campanella, C.E., Cuccovillo, N., Campanella, C., Yurt A. \& Passaro, V.M.N. (2018). Fiber Bragg grating based strain sensors: review of technology and applications, Journal of Sensors, MDPI, 18 (9):3115-.

Chapra, Steven C. (2012). Applied numerical methods with MATLAB for engineers and scientists, Third Edition. McGraw-Hill. 
Cheng, X., Xu, F., Shang, J., Li, C. (2018). A study on the amplification of active-mirror Yb:YAG lasers. Kuwait J. Sci. 45 (4) pp $46-51$.

Chow, J., Town, G., Eggleton, B., M., Ibsen, Sugden K., \& Bennion, I. (1996). Multiwavelength generation in an erbium-doped fiber laser using in-fiber comb filters. Journal of IEEE Photonics Technology Letters. 8 (1): 60-62.

Eggleton, B.J., Krug, P.A., Poladian L. \& Othonos, A. and Kalli, K. (1999). Fiber Bragg gratings-fundamental and applications in telecommunications and sensing, Pp. 97-98. Norwood, Artech House Print on Demand; Illustrated edition.

Eggleton B.J., Krug P.A., Poladian L., \& Ouellette, F. (1994). Long periodic superstructure Bragg gratings in optical fibers. Journal of Electronics Letters, 30 (19):1620-1622.

Fdhala, M. and Khorsheed S., (2020). Characterization of uniform FBG sensor operation for signal filtering application. European Journal of Advances in Engineering and Technology $7(2): 1-6$.

Filho, A., Sales, J.C., Sousa, J.R., Freire, M.M., Alves, G.I., Sombra, S. (2019). Sensors of encoding and decoding based on superstructures fiber Bragg gratings modulated in amplitude and phase for applications in systems OCDMA-63 chip. Journal of fiber and integrated optics, 6(38): 349-361.

Hemed, A.A. and Abbas, R.S. (2020). Enhanced emission perturbations associated with mixed optical injection in laser diode. AIP Conference Proceedings 2290, (050021):1-12.

Hemed, A.A. (2011). Chaos generation methods for optical communication systems. Ph.D. dissertation, University of Baghdad, Baghdad, Iraq.

Hemed, A.A. (2005). Studying the effect of modal birefringence (high and low) in a single mode optical fiber. M.Sc. thesis, Mustansiriyah University, Baghdad, Iraq.

Hemed, A.A., Ghayib, Z.R. and Rashid, H.G. (2021). Controlling a chaotic antisynchronized oscillator by a phase interplayed optical injected seed with an FBG sensor. IOP Journal of Physics: Conference Series (1963) (012063).

Hill, K.O., Fujii, Y., Johnson, D.C. \& Kawasaki B., (1978). Photosensitivity in optical fiber waveguides: Application to reflection filter fabrication. Journal of AIP Applied Physics Letters 32(647). 
Hill, K.O., Malo, B., Bilodeau, F., Johnson, D.C., \& Albert, J. (1993). Bragg gratings fabricated in monomode photosensitive optical fiber by UV exposure through a phase mask. Appl. Phys. Lett. 62, 1035

Ibsen, M., Eggleton, B. J., Sceats, M. G., \& Ouellette, F. (1995). Broadly tunable DBR fiber laser using sampled fiber Bragg gratings. Journal of Electronics Letters, 31 (19): 1620 - 1622.

Jinsong, L., Zhang, W., \& and Williams, J.A.R. (2004). Optimization of superstructured fiber Bragg gratings for microwave photonic filters response. IEEE Photonics Technology Letters. 16(7): 1736-17-38.

Joarder, A.H. \& Omar, A.M. (2013). Exact distribution of the sum of two correlated chisquare variables and its application. Kuwait J. of science 40(2): 61-81.

Kashyap, R. (2009). Fiber Bragg grating- Second edition - Chapter 10, Pp. 441-442. Academic Press-Elsevier.

Khorsheed, S.M., Hemed, A.A. \& Fdhala, M.M. (2019). Investigation of performance for a two regions superstructure fiber Bragg grating. World Scientific News, 137:42-57.

Macrae, C.D., Bongs, K. \& Holynski, K. (2020). Optical frequency generation using fiber Bragg grating filters for applications in portable quantum sensing. Physics. Atom-ph.

Mohammed, A.Z., Abass, A.K., Ibrahim, S.K. \& Nassir, W.Y. (2018). Theoretical analysis of fiber Bragg grating tunable filter utilizing tensile /compression technique. Diyala Journal of Engineering Sciences, 11(2: 55-59.

Raphael, N. G. and de Sterke, C. Martijn (1997). Theory of grating superstructures. Journal of Physical Review E. 55: 3634-3646.

Sengupta, S. \& Ghorai, S.K. (2015). An efficient method for determining transmission characteristics of superstructure fiber Bragg grating and its use for multiparameter sensing. Optical Fiber Technology 21:146-153.

Leng, J.S., Zhang, W. \& Williams, J. A. R. (2004). Optimization of Superstructured fiber Bragg gratings for microwave photonic filters response. IEEE Photonics Technology Letters, 16(7): $1736-1738$.

Panga, B. , Gu, Z., Ling, Q., Wu, W. \& Zhou, Y. (2020). Simultaneous measurement of temperature and surrounding refractive index by superimposed coated long period fiber grating and fiber Bragg grating sensor based on mode barrier region. Science direct, Journal of Optik, Elsevier GmbH, 220. 
Woyessa, G., Pederson, J. K., Nilson, K.Sengupta, S., Ghorai S. K., \& Biswas, P. (2016).Design of superstructure fiber Bragg grating with efficient mode-coupling for simultaneous strain and temperature measurement with low cross-sensitivity. IEEE Sensors Journal. 16(22): 7941-7949.

Ouellette (1994). Long periodic superstructure Bragg gratings in optical fibers. Electronics Letters 30(19): 1620-1622.

Singh, A. K., Berggren, S., Zhu, Y., Han, M. \& Huang, H. (2017). Simultaneous strain and temperature measurement using a single fiber Bragg grating embedded in a composite laminate. Smart Materials and Structures-IOP Publishing. 26: 115025, 10Pp.

Tan, R.X., Yap, S.H.K., Tan Y.C., Tjin, S.C., Ibsen, M., Yong, K.T. \& Lai, W. J. (2018). Functionalized fiber end superstructure fiber Bragg grating refractive index sensor for heavy metal ion detection. Sensors MDPI. 18(6):1821-.

Tosi, D. (2017). Improved KLT algorithm for high-precision wavelength tracking of optical fiber Bragg grating sensors. Hindawi Journal of Sensors, article ID 5412825, 10 pages.

Zhang, A-P., Tao, X-M., Chung, W-H., Guan, B-O., \& Tam H-Y. (2002) Cladding-modeassisted recouplings in concatenated long-period and fiber Bragg gratings. Optics Letters 27(14): 1214-1216.

Zhao, J. (2001). An object-oriented simulation program for fiber Bragg grating, M.Sc. thesis, Rand Africans University, Johannesburg, South Africa.

Submitted: $20 / 02 / 2021$

Revised: $\quad 13 / 10 / 2021$

Accepted: 14/03/2021

DOI: $10.48129 / \mathrm{kjs} . v 49 \mathrm{i} 1.12487$ 